\title{
Article
}

\section{Type 1 diabetes mellitus induces structural changes and molecular remodelling in the rat kidney}

\author{
Singh, Jaipaul
}

Available at http://clok.uclan.ac.uk/21638/

Singh, Jaipaul ORCID: 0000-0002-3200-3949 (2018) Type 1 diabetes mellitus induces structural changes and molecular remodelling in the rat kidney. Molecular and Cellular Biochemistry . ISSN 0300-8177

It is advisable to refer to the publisher's version if you intend to cite from the work. http://dx.doi.org/10.1007/s11010-018-3338-4

For more information about UCLan's research in this area go to http://www.uclan.ac.uk/researchgroups/ and search for <name of research Group>.

For information about Research generally at UCLan please go to http://www.uclan.ac.uk/research/

All outputs in CLoK are protected by Intellectual Property Rights law, including Copyright law. Copyright, IPR and Moral Rights for the works on this site are retained by the individual authors and/or other copyright owners. Terms and conditions for use of this material are defined in the policies page.

\section{CLoK}

Central Lancashire online Knowledge www.clok.uclan.ac.uk

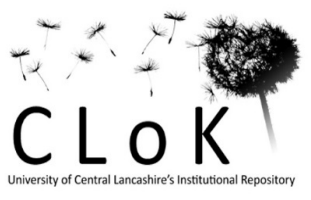




\section{Type 1 diabetes mellitus induces structural changes and molecular remodelling in the rat kidney}

Raphael M. Singh ${ }^{1,2}$, Frank C. Howarth ${ }^{3}$, Ernest Adeghate ${ }^{4}$, Keshore Bidasee $^{5}$, Jaipaul Singh ${ }^{1}$ and Tehreem Waqar ${ }^{1}$

${ }^{1}$ School of Forensic and Applied Sciences, University of Central Lancashire, Preston, PR1 2HE, England, United Kingdom, ${ }^{2}$ Faculty of Medicine and Health Sciences, University of Guyana, Turkeyen, Georgetown, Guyana, ${ }^{3}$ Department of Physiology and ${ }^{4}$ Department of Human Anatomy, College of Medicine and Health Sciences, United Arab Emirates University, AL-AIN, United Arab Emirates and ${ }^{5}$ Department of Pharmacology and Experimental Neurosciences, University of Nebraska Medical Centre, Omaha, Nebraska, 68198, United States of America.

Running Title: Diabetes-induced nephropathy

\section{Corresponding author}

Corresponding Author: Raphael M. Singh, Faculty of Medicine and Health Sciences, University of Guyana, Turkeyen, Georgetown, Guyana. (Email: raphael_singh@yahoo.com Telephone: $+5926285115)$ 


\begin{abstract}
There is much evidence that diabetes mellitus (DM) -induced hyperglycemia (HG) is responsible for kidney failure or nephropathy leading to cardiovascular complications. Cellular and molecular mechanism(s) whereby DM can damage the kidney is still not fully understood. This study investigated the effect of streptozotocin (STZ)-induced diabetes (T1DM) on the structure and associated molecular alterations of the isolated rat left kidney following 2 and 4 months of the disorder compared to the respective age-matched controls. The results revealed hypertrophy and general disorganized architecture of the kidney characterized by expansion in glomerular borders, tubular atrophy and increased vacuolization of renal tubular epithelial cells in the diabetic groups compared to controls. Electron microscopic analysis revealed ultrastructural alterations in the left kidney highlighted by an increase in glomerular basement membrane width. In addition, increased caspase-3 immuno-reactivity was observed in the kidney of T1DM animals compared to age-matched controls. These structural changes were associated with elevated extracellular matrix (ECM) deposition and consequently, altered gene expression profile of ECM key components, together with elevated levels of key mediators (MMP9, integrin 5 $\alpha$, TIMP4, CTGF, vimentin) and reduced expressions of Cx43 and MMP2 of the ECM. Marked hypertrophy of the kidney was highlighted by increased atrial natriuretic peptide (ANP) and brain natriuretic peptide (BNP) gene expression. These changes also correlated with increased TGF $\beta 1$ activity, gene expression in the left kidney and elevated active TGF $\beta 1$ in plasma of T1DM rats compared to control. The results clearly demonstrated that TIDM could elicit severe structural changes and alteration in biochemical markers (remodeling) in the kidney leading to diabetic nephropathy (DN).
\end{abstract}

Key words: Diabetes-induced nephropathy, Apoptosis, fibrosis, Gene expression, Type 1 diabetes, Transforming growth factor $\beta 1$ (TGF $\beta 1$ ). 


\section{Introduction}

Diabetes-induced nephropathy (DN) is a pathological condition that can lead to cardiovascular failure resulting in death of diabetic patients [1]. It is well established that the chronic exposure of the kidney to hyperglycemic conditions favours the development of extensive structural damage [2]. DN is a micro-vascular complication leading to kidney dysfunction and end-stage renal disease (ESRD) [3]. The characteristics of DN include renal glomerular hypertrophy, basement membrane thickening, and fibrosis due to accumulation of ECM proteins and regulators $[2,4]$. Clinical features include proteinuria, albuminuria, and progressive glomerular dysfunction [5].

DN does not develop in the absence of HG, even in the presence of a genetic predisposition [6]. Nevertheless, HG is a crucial factor in the development of DN because of its effects on glomerular and mesangial cells, but alone it is not causative. Mesangial cells are crucial for maintenance of glomerular capillary structure and for the modulation of glomerular filtration via smooth-muscle activity [6]. HG is associated with an increase in mesangial cell proliferation and hypertrophy, as well as increased matrix production and basement membrane thickening. Several in vitro studies have demonstrated that HG is associated with increased mesangial cell matrix production and mesangial cell apoptosis [7]. One mechanism whereby HG can elicit unfavourable remodelling changes is via the activation of the pro-fibrotic cytokine TGF $\beta 1$. This concept is reinforced by previous experimental and clinical studies highlighting the functional linkage of HG-stimulated elevations in protein synthesis, in particular of ECM proteins with increased TGF $\beta 1$ signaling in the kidney [8]. Additionally, the role of TGF $\beta 1$ in DN has been highlighted in resultant increases in renal mesangial cells, kidney hypertrophy and cell survival [9]. TGF $\beta 1$ plays a major role in glomerular alteration in diabetic sclerosis via induction in transient actin cytoskeleton disassembly in mesangial cells, high production of fibronectin, collagen types I and IV, and mesangial cell hypertrophy. Thus, TGF $\beta 1$ may be considered as an important therapeutic target in DN.

The present study was specifically designed to investigate the duration of T1DM on the kidney employing animals induced with T1DM for 2 and 4 months compared to many previous studies 
that only investigated short- term effect of T1DM. In addition, the study attempted to ascertain whether structural remodelling in the kidney follows a similar pattern as myocardial matrix remodelling during diabetes for comparison.

\section{Methods}

Ethical Clearance: The project obtained relevant clearance from the Animal Ethics Committees at University of Central Lancashire, UK, University of Nebraska Medical Center, Omaha, NE, USA and College of Medicine \& Health Sciences, United Arab Emirates University, UAE. All procedures were in confirmation with the 'UK Animals (Scientific Procedures) Act 1986'. Animals were housed in groups ( 1 or 2 in 1 cage) under institutional regulations at standard animal housing conditions. Animals were kept at an average room temperature of $24^{\circ} \mathrm{C}, \mathrm{a}$ relative humidity of $50 \%$ and a 12 -hour day and night cycle. All rats were allowed unlimited access to water and commercial chow (unless indicated). All rats were regularly monitored throughout the experimental period for any signs of suffering or disease.

Induction of Diabetes: This study employed 24 young adult male Wistar rats, 5-6 weeks old. The rats were divided into two groups of 12 each. One group was rendered diabetic by a single intraperitoneal injection (i.p.) of streptozotocin (STZ) (Sigma-0130) (60 mg kg-1 body weight) dissolved in citrate acid buffer (0.1 M citric acid, $0.1 \mathrm{M}$ sodium citrate in distilled water, $\mathrm{pH} 4.5$ ). Control animals received an equal volume $(0.3 \mathrm{ml})$ of the citrate buffer alone. All rats were caged separately and fed routinely on a normal diet and water until after 2 months or 4 months of diabetes, when they were humanely killed for experimentations. DM was confirmed 4-5 days following STZ injection and on the day, prior to killing the rats, using a glucose meter (One Touch II glucose meter, Lifescan inc).

Sample collection: Wistar rats were humanely killed by a blow to the head followed by cervical dislocation and the left kidney rapidly removed by mid-abdominal incision. Blood samples were taken directly from the heart by cardiac puncture. Following the removal of the kidney, it was blotted dry, weighed and either frozen in liquid $N_{2}$ for protein assay/gene expression and stored at $-80^{\circ} \mathrm{C}$ or processed for light/electron microscopy. For all animals, body weight and 
kidney weight were measured immediately after isolation and non-fasting blood glucose was measured prior to experiments.

\section{Histological studies}

Tissues were processed for histological examination according to previously described techniques [10-11]. Briefly, kidney tissue samples were fixed in $10 \%$ formalin and dehydrated in alcohol series for specific time limits. Paraffin-embedded samples were sectioned at $4 \mu \mathrm{m}$ using HM325 microtome, deparaffinized, rehydrated and stained with hematoxylin and eosin (H\&E) for general examination and with Masson's trichrome for the determination of ECM deposition according to previously established methods [10-11]. Morphometry was performed by digital image analysis using a PC digital image camera (Digital Sight DS-5M, Nikon Corp, Japan) mounted on an Axiolab Zeiss light microscope (Carl Zeiss Corp, Germany) with a 40X objective. Extent of ECM proliferation in the LV and Kidney was assessed using Image J software (National Institutes of Health, Bethesda, MD, USA; http://www.nih) and colour segmentation plugin was applied to calculate the percentage of interstitial fibrosis present in the Masson's trichrome stained kidney sections. Image J was programmed to identify shades of a specific colour (green for collagen) on the stained sections. Approximately, 30-40 selected fields in each group were included in the analysis.

\section{Immuno-histochemistry}

The activity of caspase- 3 as a primary effector of kidney tissue apoptosis was measured using a commercially available caspase-3 detection kit following the manufacturers' instructions (Signal-Stain Apoptosis (Cleaved Caspase-3) IHC detection kit, Cell Signaling Technology, 12692). Briefly, paraffin-embedded sections were deparaffinised a series of alcohol before antigen retrieval. Sections were then blocked with 1x TBST/5\% normal goat serum and incubated for 1 hour at room temperature. Sections were incubated over night at $4^{\circ} \mathrm{C}$ with polyclonal rabbit anti-active caspase 3 antibody diluted in antibody diluent (1:500) supplied within the kit. $30 \mu l$ of chromagen concentrate was added to $1 \mathrm{ml}$ of Signal Stain DAB Diluent, which was then applied to each section and monitored closely for acceptable staining intensity (red-brown) 
monitored by light microscopy. A semi-quantitative analysis of the apoptotic cells that was on average of 30-40 LV groups, defined as caspase-3 positive cells/area $\left(\mathrm{mm}^{2}\right)$ were obtained using Image J. Photomicrographs were imaged at X400.

\section{Electron Microscopy}

Randomly selected semi-thin sections, were cut with glass knives and stained with Toluidine blue for examination by light microscopy in order to exclude abnormal tissues from

ultrastructural studies. Thereafter, semi-thin sections were stained with methylene blue and examined in the light microscope to select appropriate areas for the preparation of ultrathin sections. Ultrathin sections were cut with a diamond knife (DuPont) on LKB III ultratome and picked up on mesh copper grids. Sections were carefully stained with saturated uranyl acetate solution in 50 percent ethanol for 15 min followed by lead citrate solution for two min. A JEM 1010 electron microscope was used to examine the ultrathin sections at an accelerating voltage of $60 \mathrm{kV}$. Subsequently, electron micrographs were printed on $8 \times 10$ inch photographic paper at 7,000 $\mathrm{X}$ and $14,000 \mathrm{X}$ magnifications.

\section{mRNA quantification by Quantitative Reverse transcriptase Polymerase chain reaction: mRNA} isolation and generation of cDNA

A two-step SYBR green I RT-PCR protocol was used to quantify mRNA expression of numerous target genes in 'real time' RTqPCR assays. The primer sequences, experimental conditions, design and techniques detailed below had previously been validated [13]. Briefly, frozen kidney samples were cut into $10 \mu \mathrm{m}$ sections using a cryostat, making sure the samples do not thaw. Total RNA was isolated from sections using Qiagen muscle RNA extraction procedure and manufacturer's instructions (Qiagen, Hilden, Germany). Briefly, $10 \mu \mathrm{l}$ of $\beta$-mercaptoethanol was added to $1 \mathrm{ml}$ of 'RLT' buffer provided and a stock of carrier RNA was prepared. Samples were homogenized and a volume of $5 \mu$ l of proteinase $\mathrm{K}$ was then added to digest proteins in tissue. A second elution gave a final volume of approximately $90 \mu$ that was left to precipitate overnight at $-20^{\circ} \mathrm{C}$ followed by centrigugation, and resulting RNA was ethanol precipitated and pellets were dissolved in $10 \mu \mathrm{l}$, RNAse free water. Thereafter, the concentration and purity of 
RNA samples was measured at A260 and the ratio of absorbance at 260 and $280 \mathrm{~nm}$ with a Nanodrop ND-1000 spectrophotometer (Nanodrop Techologies, USA).

A volume of $10 \mu \mathrm{l}$ of the RT/buffer master mix was added while samples were on ice. Samples were then incubated at $25^{\circ} \mathrm{C}$ for $10 \mathrm{~min}$ in the PCR machine, for first priming, $50^{\circ} \mathrm{C}$ for $50 \mathrm{~min}$, for further synthesis and $85^{\circ} \mathrm{C}$ for $15 \mathrm{~min}$, to stop the reaction. Aliquots of neat cDNA were diluted ( $2 \mu$ into $18 \mu \mathrm{l} \mathrm{H}_{2} \mathrm{O}$ ) giving a 1:10 ratio for direct use in qPCR.

\section{QPCR}

Gene expression analyses were performed by reverse transcription and qPCR. RTqPCR was achieved using an ABI Prism 7900 HT Sequence Detection System (Applied Biosystems, Foster, USA). The size of QPCR products and specificity of primers were tested in the laboratory previously by running samples on $2 \%$ agarose gels containing ethidium bromide and visualized by UV light. For this study commercially available primers were utilized which gave amplicons of similar size. The reaction mixture consisted of $1 \mu$ of cDNA, 1x Qiagen assay, 1x SYBR Green Master Mix (Applied Biosystems, 4367659) and the final volume of $10 \mu \mathrm{l}$ was made up with DNAse-free water.

Gene expression was analyzed using SDS 2.1.1 software (Applied Biosystems) by a double standardization method known as the modified $2^{-\Delta \Delta C t}$ that was adjust for PCR efficiency differences [14]. Glyceraldehyde-3-phosphate dehydrogenase (GAPDH) and 18S ribosomal RNA were analyzed to determine the housekeeping gene whose amplification efficiency is equal to other PCR targets of this study. Ratios relative to the calibrator were determined based on the respective delta cycle tresholds. Expression levels were articulated as value of mRNA abundance relative to average GAPDH content.

\section{Transforming growth factor $\beta 1$ Immunoassay}

Protein extraction - Left kidney samples from STZ-induced T1DM rats for 2 and 4 months and Wistar age-matched control animals were homogenized in in homogenization buffer, $p \mathrm{H} 7.4$ as recommended by ELIZA kit manufacturers. Sample homogenate was sonicated and centrifuged 
at $14,000 \mathrm{~g}$ for $1 \mathrm{~min}$ at $4{ }^{\circ} \mathrm{C}$. The supernatant was collected and aliquoted into tubes and further assayed for protein concentration or stored at $-80^{\circ} \mathrm{C}$.

Modified Lowry protein assay - Protein concentrations in tissue homogenates of the kidney were assayed employing the modified Lowry method previously described [14] and a microplate reader (Micro Quant, Biotech Instruments) was used for analysis. The linearity range for protein detection was given as $1-1,500 \mu \mathrm{g} / \mathrm{ml}$.

Total and active Transforming growth factor $\beta_{1}$ detection by Enzyme-Linked ImmunoSorbant Assay (ELIZA) - Total and biologically active TGF $\beta 1$ protein in serum and from frozen samples was measured using an enzyme-linked immunosorbent assay (ELISA) kit (TGF $\beta 1$ Emax ImmunoAssay System, Promega, USA) and manufacturer's instructions.

Detection of endogenous TGF $\beta_{1}$ in plasma, LV and kidney homogenates (extracted as mentioned above) from aged matched control and STZ-induced and GK subgroups were achieved using manufacturer's protocol.

Sample preparation was achieved by dividing plasma and kidney homogenates in 2 equal fractions to assay for total and active $T G F \beta_{1}$. A volume of $100 \mu$ of either acid treated or naturally processed samples were added to the coated plate and incubated for 90 minutes at room temperature with shaking $(500 \pm 100 \mathrm{rpm})$. Samples were diluted in buffer prepared according to manufacturer protocol. After washing, the specifically bound polyclonal antibody was detected by diluting $10 \mu \mathrm{l}$ stock Anti-TGF $\beta_{1}$ pAb in $10 \mathrm{ml}$ of Sample $1 \mathrm{X}$ buffer (1:1000). A volume of $100 \mu \mathrm{l}$ of this was pipetted in each well and incubated for 2 hours at room temperature with shaking $(500 \pm 100 \mathrm{rpm})$. Samples were incubated with $100 \mu$ of TMB One Solution (chromagen substrate) for 15 minutes at room temperature, which produced a blue colour in the wells. The reaction was stopped using $100 \mu \mathrm{l}$ of $1 \mathrm{~N} \mathrm{HCl}$, which changed the colour to yellow and was measured at $450 \mathrm{~nm}$ using a microplate reader (Anthos Hill, Biochrome Ltd, UK). Samples were analyzed in duplicate and the results were obtained by extrapolation from the TGF $\beta_{1}$ standard curve and expressed as ratio of Active: Total TGF $\beta_{1}$ for plasma samples and $\mathrm{pg} / \mathrm{mg}$ of total protein for LV and kidney homogenates. 


\section{Statistical analysis}

Quantitative data acquired from these experiments were statistically analyzed using different statistical tests. Mean $( \pm S E M)$ data were plotted as graphs with $n=3-6$ different experiments. Statistical significance of differences between age-matched control and STZ-induced diabetic data were compared by Independent samples Student's t-test using IBM SPSS statistics 22 software (SPSS Inc., Chicago, IL, USA). Unless stated otherwise, statistical comparisons were performed using one-way ANOVA followed by Bonferroni corrected t-tests for multiple comparisons. A value of $p<0.05$ was taken as statistically significant and values $<0.01$ were taken as highly significant.

\section{Results}

\section{Characteristic evaluation of the experimental model}

General characteristics of the STZ-treated T1DM rats and age-matched Wistar control are shown in Table 1. The results show that diabetes can significantly $(p<0.05)$ reduce blood glucose level and body weights at 2 and 4 months compared to the respective age-matched controls. In contrast, T1DN significantly $(p<0.05)$ increases kidney weight and kidney to body weight ratio compared to the respective controls. Changes in these different parameters were more severe following 4 month of T1DM compared to 2 months.

\section{Histopathology of the Kidneys after 2 and 4 months of STZ-treatment}

Figure 1 shows the histopathology of the left kidney at 2 and 4 months after STZ-induction compared to age-matched controls. Kidney optical analysis using the $H$ \& $E$ stain by light microscopy revealed general disorganized architecture of the kidney characterized by expansion in glomerular borders, tubular atrophy and increased vacuolization of renal tubular epithelial cells in the STZ-induced diabetic group. In contrast, the control groups revealed organized intact glomeruli, Bowman's capsule and tubular structure. 
Table 1: General characteristics of T1DM and control rats. Data are shown as the Mean ( \pm S.E.M), ${ }^{*} p<0.05{ }^{* *} p<0.01$, unpaired Student's t-test. Numbers in bracket indicate number of animals used for experiments.

\begin{tabular}{|c|c|c|c|c|}
\hline Animals & $\begin{array}{c}\text { Fasting } \\
\text { Blood } \\
\text { Glucose } \\
\text { (mg/dl) }\end{array}$ & $\begin{array}{c}\text { Body Weight } \\
\mathbf{( g )}\end{array}$ & $\begin{array}{c}\text { Kidney weight } \\
\text { (g) }\end{array}$ & $\begin{array}{c}\text { Kidney Weight/Body Weight } \\
\text { ratio (g/100g body weight) }\end{array}$ \\
\hline $\begin{array}{c}\text { Control (n=6) } \\
\mathbf{2} \text { months }\end{array}$ & $98 \pm 3.79$ & $343 \pm 9.99$ & $3.25 \pm 0.12$ & $0.94 \pm 0.01$ \\
\hline $\begin{array}{c}\text { Diabetic (n=6) } \\
\mathbf{2} \text { months }\end{array}$ & $443 \pm 12.2^{* *}$ & $270 \pm 5.78^{* *}$ & $4.22 \pm 0.13^{* *}$ & $1.56 \pm 0.02^{* *}$ \\
\hline $\begin{array}{c}\text { Control (n=8) } \\
\mathbf{4} \text { months }\end{array}$ & $97 \pm 3.04$ & $378 \pm 10.63$ & $3.08 \pm 0.13$ & $0.82 \pm 0.01$ \\
\hline $\begin{array}{c}\text { Diabetic (n=8) } \\
\mathbf{4} \text { months }\end{array}$ & $446 \pm 18.8^{* *}$ & $221 \pm 13.02^{* *}$ & $4.45 \pm 0.16^{* *}$ & \\
\hline
\end{tabular}

\section{Examination of the glomerular basement membrane}

Figure 2 shows glomerular basement membrane examination, highlighted by Periodic Acid Schiff (PAS) stained sections in the left kidney at 2 and 4 months after STZ-induction compared to age matched controls. Light microscopy revealed thickened glomerular basement membranes in both STZ-treated groups compared to age-matched control groups. Increased mesengial expansion is clearly visible in both groups as the earliest morphological change in diabetic nephropathy due to increased deposition of the mesangial matrix together with a mild increase in mesangial cellularity, and mesangial cells hypertrophy. 
Figure 1: Kidney pathology Representative light photomicrographs detailing kidney histopathology in H \& E stained kidney sections at 2 and 4 months of STZ-induced diabetes and age-matched controls. General glomeruli are intact and organized in structure. Original magnification X400. Photomicrographs are typical of 20-25 fields/per group consisting of 6-9 animals per group. Scale bar in the right hand corner is $20 \mu \mathrm{m}$ and for all micrographs. D: Distal tubulus, P: Proximal tubulus, G: Glomerulus, BC: Bowman's capsule, Tailed arrow: expanded g lomerulus borders in DM group, Block arrow: RBC that make statis in glomerulus and interstisium, Diamond; clear cells.

Figure 2: Glomerular basement membrane: Representative light micrographs of PAS stained kidney sections from 2 and 4 months after STZ-treatment. Glomerular basement membrane is 
seen to be increased in STZ-treated kidneys in comparison to age-matched controls. Original Magnification X400, Photomicrographs are typical of 35-47 fields/experimental groups consisting of 6-9 animals per group. Scale bar indicates $20 \mu \mathrm{m}$. D; Distal tubulus, P; Proximal tubulus, Tailed arrow; thickened basement membrane of glomerulus, Block arrow; increased Bowman's space, Diamond; clear cells of tubulus Triangle; Dilated tubulus.

\section{Ultrastructural study of the kidneys after 2 and 4 months of STZ-treatment}

Figure 3 shows electron microscopic images of the left kidney at 2 (A) and 4 (B) months after STZ-induction compared to age-matched controls. The image shows ultrastructural abnormality in diabetic nephropathy as seen as diffused thickening of the GBM. In addition, a variable degree of mesangial expansion by extracellular matrix deposition and increased mesangial cellularity is also present in the diabetic kidney of both groups. A reduction in podocyte number (podocytopenia), along with a reduced podocyte per glomerulus ratio can also be seen in both groups after STZ-induction. Diffuse foot process effacement and podocyte detachments are another hallmark of DN and they are visible in both STZ-treated groups. Figure $2 \mathrm{C}$ shows quantitative assessment of GBM width using Image J analysis tool. The data revealed significant $(p<0.05)$ increases at 2 months $(510 \pm 1.35$ vs. $330 \pm 1.15 \mu \mathrm{m})$ and 4 months after STZ-treatment $(540 \pm 1.50$ vs. $348 \pm 1.15 \mu \mathrm{m})$ compared to age-matched controls. 
Figure 3: Kidney Ultrastructure: Representative TEM electron micrographs showing increased mesangial matrix and glomerular basement membrane (GBM) together with increased detachment of podocytes in the kidneys from 2 and 4 months after STZ-treatment (A) and quantitative assessment of GBM $(\mathrm{nm})$ using Image J analysis tool (B). Original magnification $X$ 14,000 . Electron micrographs are representative of 35-45 photomicrographs studied from 5-6 animals per group. Scale bar in the lower right indicates $1 \mu \mathrm{m}$; MM; Mesangial Matrix, Block arrow; Thick GBM, NP; Normal Podocyte, Chevron; Podocyte detachment. Data are Mean \pm SEM, $* P<0.05$, One-way ANOVA followed by Bonferroni corrected t-tests for multiple comparisons.

\section{Kidney fibrosis after 2 and 4 months of STZ-treatment}

Figure 4 shows the results from light microscopy photomicrographs of Masson's Trichrome stained sections in the left kidney after STZ-induction compared to age-matched controls following 2 (A) and 4 months (B). STZ-treated rats demonstrated significantly increased ECM deposition in both glomerulus and tubulus regions. Figure 4C shows quantitative analysis of fibrosis in STZ-treated rats and the data revealed significantly greater glomerular area coverage as compared to age-matched controls occupying at 2 months $(5.89 \pm 0.51 \%$ vs. $2.41 \pm 0.44 \%$; $p<0.05)$ and 4 months $(7.66 \pm 0.53 \%$ vs. $3.36 \pm 0.46 \% ; p<0.01$ post STZ-treatment. These changes were significantly $(p<0.05)$ different between 2 and 4 months of STZ-treatment time indicating the severity of the diabetes in the kidney. 
Figure 4: ECC deposition in the kidney::Representative light micrographs of Masson's Trichrome stained myocardial sections from 2 months after STZ-treatment (A) and 4 months after STZ-treatment (B) and quantitative assessment of interstitial fibrosis using Image $\mathrm{J}$ analysis tool (C). Original Magnification X400, Photomicrographs are typical of 35-47 fields/experimental group consisting of 6-9 animals per group. Scale bar indicates $10 \mu \mathrm{m}$. Data are Mean $\pm \mathrm{SEM},{ }^{*} p<$ $0.05, * * p<0.01$ One-way ANOVA followed by Bonferroni corrected t-tests for multiple comparisons.

\section{Apoptosis in the kidneys after 2 and 4 months of STZ-treatment}

Figure 5 shows a significant $(p<0.05)$ increase in the immune-reactivity of cleaved (active) caspase-3 within the left kidney of STZ-treated diabetic rats compared to age-matched controls at (A) 2 months $(8.96 \pm 0.89$ vs. $1.89 \pm 0.39)$ positive cells $/ \mathrm{mm}^{2} \quad(p<0.05)$ and (B) 4 months (9.66 \pm 1.00 vs $2.49 \pm 0.50)$ positive cells $/ \mathrm{mm}^{2}$ after STZ-treatment. Figure $5 \mathrm{C}$ shows quantitative analysis of positive caspase-containing cells in the kidney of age- matched control and diabetic rats. This increase was significant $(p<0.05)$ in diabetic kidney compared to control. 
Figure 5: Kidney Apoptosis: Active Caspase-3 positive cells (brown staining) in the kidneys from 2 months after STZ-treatment (A) and 4 months after STZ-treatment (B) and quantitative assessment of immune-reactivity using Image J (C). Photomicrographs are typical of 15-20 fields/experimental groups consisting of 5-6 animals per group. Diamond; clear cells of tubulus, Block arrow; Thickened basement membrane and small glomerular size. Scale bar indicates 20 $\mu \mathrm{m}$ and for all photomicrographs. Data are Mean \pm SEM, ${ }^{*} p<0.05$, One-way ANOVA followed by Bonferroni corrected t-tests for multiple comparisons.

\section{Molecular events underlying structural remodeling in the kidneys}

\section{Gene expression for ECM components}

Figure 6 represents gene expression of the ECM components, collagen $1 \alpha$, collagen $3 \alpha$, fibronectin and elastin quantified using qRT-PCR. The data revealed a significant $(p<0.01)$ increase of collagen $1 \alpha$ after 2 months $(0.89 \pm 0.14$ vs. $0.53 \pm 0.10$ ratio units) and 4 months $(1.23 \pm 0.13$ vs. $0.58 \pm 0.11$ ratio units) of STZ-treatment (figure $6 \mathrm{~A})$. Similarly, there was a significant $(p<0.05)$ increase in collagen $3 \alpha$ after 2 months $(1.00 \pm 0.12$ vs.0.61 \pm 0.09$)$ and 4 months ( $1.45 \pm 0.13$ vs. $0.68 \pm 0.07$ ratio units) (figure $6 \mathrm{~B}$ ) in of STZ-induced diabetes compared to age-matched control. Both type of collagens showed significant $(p<0.05)$ differences between the two age- groups indicating that collagen expressions are changed over time possibly due to the duration of diabetes. Fibronectin (figure 6C) and elastin (Figure 6D) were significantly increased at 2 months (fibronectin; $1.26 \pm 0.14$ vs. $0.92 \pm 0.07$ ratio units), (elastin; $0.98 \pm 0.13$ vs. $0.55 \pm 0.05$ ratio units) and 4 months (fibronectin; $1.59 \pm 0.11$ vs. $0.92 \pm 0.07$ ratio units), (elastin; 
$1.09 \pm 0.12$ vs. $0.59 \pm 0.08$ ratio units) after STZ-induction compared to age-matched control. However, only fibronectin revealed an adult vs young adult effect that was significantly $(p<0.05)$ evident. Furthermore, these changes were accompanied by divergent alterations in ECM regulators.

Figure 7 shows the gene expression of ECM regulators MMP2, MMP9, TIMP4, CTGF, connexin 43, integrin 5 $\alpha$ and vimentin. Kidney mRNA gene expression for MMP2 (Figure 7A) revealed a significant $(p<0.05)$ down regulation at 2 months $(0.56 \pm 0.12$ vs. $1.00 \pm 0.11$ ratio units $)$ and 4 months $(0.58 \pm 0.13$ vs. $1.13 \pm 0.10$ ratio units) after STZ-induction compared to age-matched control. On the other hand, MMP9 (Figure 7B) was significantly $(p<0.05)$ up-regulated at 2 months ( $1.16 \pm 0.11$ vs. $0.86 \pm 0.02$ ratio units) and 4 months ( $1.31 \pm 0.12$ vs. $0.91 \pm 0.10$ ratio units) after STZ-induction compared to age-matched controls. Interestingly, endogenous MMP tissue inhibitor TIMP4 (Figure 7C) was up regulated at 2 months (1.00 \pm 0.12 vs. $0.67 \pm 0.06$ ratio units) and 4 months $(1.32 \pm 0.11$ vs. $0.93 \pm 0.05$ ratio units) after STZ-induction compared to agematched control and this difference was significantly $(p<0.05)$ evident between the two age groups. mRNA gene expression level for CTGF (Figure 7D) was significantly $p<0.05$ ) increased at 2 months $(1.11 \pm 0.14$ vs. $0.57 \pm 0.04$ ratio units) and 4 months $(1.23 \pm 0.15$ vs. $0.79 \pm 0.08$ ratio units) after STZ-induction compared to age-matched controls. Additionally, a significant $(p<0.05)$ down-regulation in gap junction protein CX43 (Figure 7E) was evident in the STZinduced diabetic rat kidney after 2 months (0.89 \pm 0.12 vs. $1.28 \pm 0.06$ ratio units) and at 4 months $(p<0.01)(0.45 \pm 0.14$ vs. $1.39 \pm 0.09$ ratio units) of STZ-induction compared to age-matched control and this difference was significantly $(p<0.05)$ evident between the two age groups. Additionally, mRNA level of Integrin $5 \alpha$ (Figure 7F) was significantly $(p<0.05)$ increased at 2 months ( $1.08 \pm 0.13$ vs. $0.49 \pm 0.06$ ratio units) and 4 months ( $1.19 \pm 0.15$ vs. $0.55 \pm 0.09$ ratio units) after STZ-induction compared to age-matched control. Integrin $1 \alpha$ was also studied, but remained unaffected by the disease. Finally, mRNA level of vimentin (Figure 7G) was significantly $(p<0.05)$ up-regulated at 2 months $(1.08 \pm 0.13$ vs. $0.49 \pm 0.06$ ratio units) and 4 months (1.19 \pm 0.15 vs. $0.55 \pm 0.09$ ratio units) after STZ-induction compared to age-matched control. Taken together, gene expression profile of ECM components in the kidneys revealed marked changes that were accompanied by parallel alterations in ECM regulating agents 
(MMP2 and MMP9) and tissue inhibitor (TIMP4), gap junction proteins and vimentin expression, .An imbalance in these expressions could lead to abnormal ECM deposition which remains as one of the hallmarks of DN.

\section{ECM Components}

Figure 6: Gene expression of ECM component: Summarized data showing gene expression for ECM components Collagen 1 (A), Collagen 3 (B), Fibronectin (C) and Elastin (D) in STZ-treated and age-matched controls at 2 and 4 months post STZ-treatment. Results are representative of 8 animals/per group conducted in triplicates. RT-PCR amplification was normalised to that of GAPDH. Data are expressed as mean \pm SEM. Lines over bars indicate significance $* p<0.05$, Oneway ANOVA followed by Bonferroni corrected t-tests for multiple comparisons.

Figure 7: Gene expression of ECM regulatory components: Summarized quantitative data showing gene expression for ECM regulators MMP2 (A), MMP9 (B), TIMP4 (C), CTGF (D), Connexin $43(E)$, Integrin $5 \alpha(F)$, and Vimentin (G) in STZ-treated and age-matched controls at 2 
and 4 months post STZ-treatment. Results are representative of 8 animals/per group conducted in triplicate. RT-PCR amplification was normalised to that of GAPDH. Data are expressed as mean \pm SEM. Lines over bars with asterisk indicate significance $* p<0.05, * * p<0.01$, One-way ANOVA followed by Bonferroni corrected t-tests for multiple comparisons.

\section{Assessment of hypertrophy in kidneys}

The hypertrophic biomarkers ANP and BNP were assessed in STZ-induced and age-matched controls at 2 and 4 months post STZ-treatment. Figure 8 shows the gene expression analysis for ANP and BNP. The results revealed that ANP was significantly $p<0.05)$ up-regulated at 2 months (1.55 \pm 0.16 vs. $0.56 \pm 0.09$ ratio units) and 4 months (2.50 \pm 0.14 vs. $0.67 \pm 0.06$ ratio units) after STZ-induction compared to age-matched control. There was a significant $(p<0.05)$ difference between the two age groups, indicating a diabetes-induced age-related effect. On the other hand, BNP was significantly $(p<0.05)$ up-regulated at 4 months $(0.89 \pm 0.12$ vs. $0.55 \pm 0.09$ ratio units) after STZ-induction compared to age-matched controls. However, no significance $(p<0.05)$ was observed at 2 months $(0.55 \pm 0.13$ vs. $0.49 \pm 0.66$ ratio units $)$ after STZ-induction.

Figure 8: Gene expression for ANP and BNP biomarkers: Summarized quantitative data showing ANP (A), and BNP (B) gene expression in STZ-treated and age-matched controls at 2 and 4 months post STZ-treatment. Results are representative of 8 animals/per group conducted in triplicates. RT-PCR amplification was normalized to that of GAPDH. Data are expressed as mean \pm SEM. Lines over bars with asterisk indicate significance $* p<0.05, * * p<0.01$, One-way ANOVA followed by Bonferroni corrected t-tests for multiple comparisons.

TGF $\beta 1$ in plasma and kidney after 2 and 4 months of STZ-treatment 
Figure 9 shows plasma level as well as total, active and gene expression for TGF $\beta 1$ in the kidney. The results show that TGF $\beta 1$ was significantly $(p<0.05)$ increased in plasma after 2 months $(2.72 \pm 0.20$ vs. $1.15 \pm 0.04 \mathrm{ng} / \mathrm{ml})$ of STZ-induced diabetes compared to control whereas no significant differences were observed after 4 months $(2.36 \pm 0.06$ vs. $2.18 \pm 0.07 \mathrm{ng} / \mathrm{ml})$ of STZtreatment (Figure 9A). Total TGFB1 levels were significantly $(p<0.05)$ increased in STZ-induced diabetic kidney at 2 months (9.53 \pm 0.12 vs. $3.44 \pm 0.20 \mathrm{pg} / \mathrm{mg}$ of total protein) and 4 months (11.92 \pm 0.32 vs. $4.73 \pm 0.15 \mathrm{pg} / \mathrm{mg}$ of total protein) after STZ-treatment compared to agematched control and this significance was apparent between the two age groups (Figure 9B). Similarly, active TGF $\beta 1$ levels in kidneys were significantly $(p<0.01)$ increased after 2 months (60.75 \pm 0.38 vs. $28.83 \pm 0.35 \mathrm{pg} / \mathrm{mg}$ of total protein) and 4 months (59.68 \pm 0.32 vs. $36.33 \pm 0.28$ $\mathrm{pg} / \mathrm{mg}$ of total protein) of STZ-induction compared to age-matched control (Figure 9C). However, these differences were not significantly different between the two age groups. TGF $\beta 1$ gene expression was also significantly $(p<0.05)$ different between the two age groups (after 2 months; (1.25 \pm 0.13 vs. $0.55 \pm 0.05)$ and after 4 months; $(1.79 \pm 0.15$ vs. $0.88 \pm 0.08)$ in STZ induced diabetic rat LV compared to age-matched control this significance was apparent between the two age groups (Figure 9D).

Figure 9: Plasma level, total, active and gene expression of TGF $\beta 1$ : Summarized quantitative data from ELIZA showing ratio of active : total TGF $\beta 1$ protein in plasma (A), total TGF $\beta 1$ : total extracted kidney protein (B) and active TGF $\beta 1$ : total extracted kidney protein (C) at 2 and 4 months of STZ-treatment with age-matched control animals ( $n=3 /$ group). mRNA gene expression of TGF $\beta 1$ (D) at 2 and 4 months of STZ-treatment with age-matched controls. The results of both ELIZA and qRT-PCR are representative of 3 individual experiments conducted in triplicate. RT-PCR amplification was normalized to that of GAPDH. Data are expressed as mean \pm SEM. Lines over bars indicate significance * $p<0.05, * * p<0.01$, One-way ANOVA followed by Bonferroni corrected t-tests for multiple comparisons. 


\section{Discussion}

In this study, the morphological and molecular consequences of HG-induced diabetes have been characterized in the left kidney of STZ-induced type 1 diabetics male rats compared to age-matched controls. The left kidney is slightly larger than that of right kidney and it is positioned slightly upper than the right kidney due to asymmetry within the abdominal cavity caused by the liver. In addition, its posterior surface is associated with the spleen, pancreas, stomach and other organs surrounding it. Male rats are normally employed in most diabetic studies in order to avoid any complications of other hormonal changes that may occur in females, but not in male animals. The discussion will now focus on the various measured parameters in this study.

Firstly, the general characteristics of rats revealed significant decreases in body weight in STZinduced rats compared to the respective controls. In contrast, there were marked elevations in blood glucose levels, kidney weights and kidney weight to body weight ratios observed in the STZ-induced rat at 2 and 4 months after diabetes-induction compared to control. The increase in kidney size is an indication of renal hypertrophy, which is the hallmark of DN [15-16]. This increase in size is primarily due to glomerular and tubular hypertrophy, although some lowgrade proliferations of glomerular cells are also present in the early stage of DN $[16,17]$. Glomerular hypertrophy is in part the result of glomerular cell hypertrophy and recent studies have suggested that the diabetic milieu per se, hemodynamic changes and local growth factors such as TGFB1 and angiotensin II (ANG II) are mediators in the pathogenesis of glomerular cell hypertrophy [16]. The results of this study are consistent with previously published reports on renal hypertrophy [18]. Previous in vitro studies have reported that high glucose, TGF $\beta 1$ and ANG II are mediators of DN and they are closely linked with the Akt pathway [19]. Owing to these findings, the activity of Akt under diabetic conditions has been heavily investigated, but discrepancies remain, some have demonstrated an increase in Akt phosphorylation [19].

The results of this study revealed marked elevations in gene expression levels for ANP and BNP, which are sensitive biomarkers for hypertrophy. The up-regulation in ANP and BNP levels in the left kidney at 2 and 4 months post STZ-induction compared to age-matched controls is 
consistent with previous studies [20]. Therefore, monitoring of ANP and BNP can play an important role in the management of DN.

\section{Ultrastructure studies of the kidney in T1DM}

Ultrastructural analysis employed in this study revealed an increase in GBM thickness in the kidney from 2 and 4 months of STZ-induced diabetes compared to age-matched control animals. Data from the literature suggest that GBM thickening is a characteristic of early change in T1DM [21] and T2DM-induced DN [22] and it increases with the disease duration [23]. GBM thickening is a consequence of ECM accumulation, with increased deposition of normal ECM components together with altered expression of the regulators of EM, similar to the findings of this study (Figure 6). Another hallmark feature of DN is the derangement of the architecture of podocytes and glomerular endothelial cells which participate in initiation and progression of nephropathy resulting in significant effect on the function of the kidney [24]. Podocyte detachment (PD), (Figure 5) and structural changes in DN have previously been described in T1DM with similar results to the findings of this study [24-25].

\section{Apoptosis in the diabetic kidney}

Increased apoptosis mediated by caspase-3 immuno-reactivity has been highlighted in this study. At both 2 and 4 months after TIDM induction, the rat kidney was infiltrated with increased apoptotic cells compared to respective age-matched control groups. Apoptosis has been documented in the time course of various renal diseases and more frequently in DN [26]. Cell death by apoptosis is believed to be involved in the process of mesangial cell loss in the late stage of DN [27]. In addition, apoptosis is considered to be one of the underlying causes of podocyte loss, which contributes to the development of albuminuria in DN [26]. STZ-induced diabetic rats at 2 and 4 months displayed signs of tubular, as well as, interstitial apoptosis compared to respective control groups and these results are in correlation with previously published study in STZ-diabetic rat kidney [28]. A previous study has shown that this damaging due to T1DM can be reversed by insulin therapy [28]. Moreover, Ang II type $1\left(\mathrm{AT}_{1}\right)$ and type 2 $\left(A T_{2}\right)$ Ang II receptor blockade can reduce apoptosis in the diabetic kidney indicating therapeutic relevance in the treatment of DN [29]. 


\section{Role of TGF $\beta 1$ in kidney remodeling}

The present study supports previous observations that TGFB1 is upregulated in the diabetic kidney and that this upregulation is associated with glomerulosclerosis and tubule-interstitial fibrosis [30]. Numerous studies have demonstrated that TGF $\beta 1$ promotes renal cell growth, stimulates synthesis of key ECM proteins including, type I and type II collagen, laminin and fibronectin and inhibits ECM degradation by decreasing the activity of MMPs [15]. These results in the literature are consistent with findings from this study which showed marked elevations in ECM components (Col 1 $\alpha$, Col $3 \alpha$, elastin and fibronectin) together with glomeru-sclerosis in the STZ-induced diabetic kidney at 2 and 4 months post STZ-induction compared to agematched control animals.

TGFß1 plays an important role in the pathophysiology of renal disease, including DN. Furthermore, the present study showed an overall increase in TGF $\beta 1$ activity in left kidney of 2 and 4 months after STZ-induction compared to respective age-matched controls. Elevated TGF $\beta 1$ activity was correlated with parallel findings of increased TGF $\beta 1$ gene expression that is consistent with previous findings with correlations to fibrosis in animals [31] and in humans [15]. A previous study reported that TGFB1 is over expressed in glomerular mesangial cells, proximal and distal tubules in the STZ-induced diabetic rat after 9 weeks of diabetes [32]. These authors demonstrated that supplementation with $17 \beta$-estradiol (E2) for 8 weeks following a 9week duration of diabetes attenuates TGF $\beta 1$ protein expression, suggesting that one of the mechanisms by which E2 exerts its reno-protective effects in the diabetic kidney is by regulating the expression of locally active cytokines, such as TGF $\beta 1$.

The results of this study have also revealed significant increases in the gene expression of CTGF, another pro-sclerotic cytokine, in the kidney of T1DM rats compared to the respective controls CTGF has been shown to be involved in both the early and later stages of DN. CTGF expression is seen to be increased in experimental diabetic glomerulosclerosis [33]. Elevated CTGF levels in glomeruli of NOD mice appear to correlate with the duration of diabetes [34]. Elevated CTGF expression has also been detected in human DN [33]. Gromerular CTGF levels have been found 
to be elevated in diabetic patients with micro-albuminuria as well as overt nephropathy [35]. Several in vitro studies have shown that CTGF is induced in renal cells by high glucose and AGE [36], as well as ROS [37], which they generate. CTGF is one of the TGF $\beta 1$ inducible immediate early genes and has shown to be induced by TGF $\beta 1$ [38CTGF is a crucial mediator for TGF $\beta 1$ stimulated matrix protein expression. Additionally, CTGF has been shown to mediate TGF 1 induced increases in fibronectin and collagen type I [39]. Collectively, these observations are consistent with the findings of the present study and correlate with the fact that although all these factors likely interact in the pathogenesis of DN, the driving force behind the cytokine expression is most likely to be HG, either directly or indirectly.

\section{Role of metalloproteinases in T1DM}

The results of this study have shown reciprocal changes in MMP2 and MMP9. The level of $\mathrm{MMP}_{2}$ decreased significantly in the kidney of diabetic rats compared to controls, whereas $\mathrm{MMP}_{9}$ and $\mathrm{TIMP}_{4}$ increased significantly in T1DM compared to control. MMPs are major determinants of extracellular matrix degradation and turnover in the glomerulus. Hence, the changes in either MMP expression or activity can influence intra-renal ECM composition [40]. Renal hypertrophy is prognostic of the development of micro-albuminuria in type 1 DM [41]. Since abnormal extracellular matrix deposition is the hallmark of diabetic nephropathy, it is to be expected that either altered MMP expression or activation also contributes to diabetic nephropathy, and specifically to the onset of this characteristic renal hypertrophy.

It has been reported that MMP9 was dramatically increased in the glomeruli of diabetic mice, and MMP9 deficiency attenuates diabetic nephropathy by modulation of podocyte function and dedifferentiation. Collectively, these results suggest MMP9 can play a role in the development of diabetic nephropathy [42]. Elevated MMP9 levels have strong correlations with age, body mass index (BMI) and HbA1c levels, as well as progression of diabetes [43]

Previous studies in humans have also found circulating TIMP1, TIMP 4 and $\mathrm{MMP}_{2}$ to be decreased in patients with DN [44]. Furthermore, Del Prete et al. [45] demonstrated a dramatic decrease in MMP2 gene expression in glomeruli of patients with T2DM. However, another 
study showed that $\mathrm{MMP}_{2}$ protein and related-enzyme activity were up-regulated in kidneys of patients with diabetes, as assessed by Western blot analysis and ELISA methods [46].

The MMPs have long been identified as critical mediators of ECM degradation and turnover, but increasing evidence suggests that they, in conjunction with TIMPs, play an important role in the progression of diabetic nephropathy. Additionally, TIMP4 is involved in suppressing the activity of $\mathrm{MMP}_{1}, \mathrm{MMP}_{2}, \mathrm{MMP}_{3}, \mathrm{MMP}_{7}$ and $\mathrm{MMP9}$ [47].

The present results revealed marked decreases in Cx43 levels of 2 and 4 months STZ-induced diabetic rat kidney compared to age-matched controls and this change was more severe with the duration of the diabetes. Previous studies in human kidney cells in culture have highlighted the possibility of a protective effect of $\mathrm{Cx} 43$ in preventing renal damage [48]. Specifically, studies in human collecting duct cell lines found that as glucose concentrations increased, there was a time-dependent increase in levels of $\mathrm{Cx} 43$ that is opposite to the observations made in the present study [48]. The increase in Cx43 and gap junctional communication correlated with functional acceleration of calcium transients between cells. Furthermore, levels of Cx43 have also been studied in human DN as a predictive marker of disease progression and severity [49]. Another study reported that down-regulation of Cx43 within podocytes was closely associated with disease progression in established DN and correlated with the degree of future decline in renal function [50]. Additionally, vimentin was found to be elevated in STZ-induced diabetic rat kidney at 2 and 4 months of STZ-induced DM. Vimentin is a major intermediate filament (IF) protein of the mesenchymal cells and is physiologically important as it shows dynamically altered expression patterns during different developmental stages [51].

Major functions of vimentin involve the maintenance of cellular integrity and provide resistance against stress [52]. A number of studies have found the role of TGF $\beta 1$ in response element within the activated protein complex-1 region of the vimentin promoter to be involved in regulation of vimentin expression [52]. Thus, the increased gene expression of vimentin may be closely related to the increased TGF $\beta 1$ activity observed in this study. Furthermore, this may depend on the duration of the diabetes since the expression of both TGF $\beta 1$ and vimentin increased at 4 month compared to 2 months. 


\section{Conclusion}

In conclusion, the results of this study clearly demonstrated that TIDM can elicit severe structural changes and remodelling of the kidney as revealed by the fibrosis, similar to that seen in the myocardium of diabetic rats [10-11]. These associated structural and biochemical alterations can lead to diabetic nephropathy (DN) and this in turn can be more severe depending on the duration of the diabetes. 


\section{References}

1. Gallagher H, Suckling EJ (2016) Diabetic nephropathy: where are we on the journey from pathophysiology to treatment?. Diabetes, Obesity and Metabolism 18: 641-647.

2. Decleves $A E$, Sharma K (2010) New pharmacological treatments for improving renal outcomes in diabetes. Nat Rev Nephrol 6: 371-380.

3. Kato M, Yuan H, Xu ZG, Lanting L, Li SL, Wang M, Hu MC, Reddy MA, Natarajan R (2006) Role of the Akt/FoxO3a pathway in TGF-beta1-mediated mesangial cell dysfunction: a novel mechanism related to diabetic kidney disease. J Am Soc Nephrol 17: 3325-3335.

4. Kato M, Park JT, Natarajan R (2012) MicroRNAs and the glomerulus. Exp Cell Res 318: 993-1000.

5. Kato M, Zhang J, Wang M, Lanting L, Yuan H, Rossi JJ, Natarajan R (2007) MicroRNA-192 in diabetic kidney glomeruli and its function in TGF-beta-induced collagen expression via inhibition of E-box repressors. Proc Natl Acad Sci U S A 104: 3432-3437.

6. Schena FP, Gesualdo L (2005) Pathogenetic mechanisms of diabetic nephropathy. J Am Soc Nephrol 16 Suppl 1:S30-3., S30-S33.

7. Mishra R, Emancipator SN, Kern T, Simonson MS (2005) High glucose evokes an intrinsic proapoptotic signaling pathway in mesangial cells. Kidney Int 67: 82-93.

8. Ziyadeh FN (2004) Mediators of diabetic renal disease: the case for tgf-Beta as the major mediator. J Am Soc Nephrol 15 Suppl 1:S55-7., S55-S57.

9. Deshpande SD, Putta S, Wang M, Lai JY, Bitzer M, Nelson RG, Lanting LL, Kato M, Natarajan R (2013) Transforming growth factor-beta-induced cross talk between p53 and a microRNA in the pathogenesis of diabetic nephropathy. Diabetes 62: 3151-3162.

10. D'Souza A, Howarth FC, Yanni J, Dobryznski H, Boyett MR, Adeghate, Bidasee KR, Singh J (2011) Left ventricle structural remodelling in the prediabetic Goto-Kakizaki rat. Exp Physiol 96: 875-888. 
11. D'Souza A, Howarth FC, Yanni J, Dobrzynski H, Boyett MR, Adeghate E, Bidasee KR, Singh J (2014) Chronic effects of mild hyperglycaemia on left ventricle transcriptional profile and structural remodelling in the spontaneously type 2 diabetic Goto-Kakizaki rat. Heart Fail Rev 19: 65-74.

12. Yanni J, Tellez JO, Sutyagin PV, Boyett MR, Dobrzynski H (2010) Structural remodelling of the sinoatrial node in obese old rats. J Mol Cell Cardiol 48: 653-662.

13. Skern R, Frost $P$, Nilsen $F(2005)$ Relative transcript quantification by quantitative PCR: roughly right or precisely wrong? BMC Mol Biol 6:10: 1-3.

14. Lowry OH, Rosebrough NJ, Farr AL, Randaal RJ (1951). Protein measurement with the Folin phenol reagent. J Biol Chem 193: 265-275.

15. Border WA, Yamamoto T, Noble NA (1996) Transforming growth factor beta in diabetic nephropathy. Diabetes Metab Rev 12(4): 309-339.

16. Wolf G (2004) New insights into the pathophysiology of diabetic nephropathy: from haemodynamics to molecular pathology. Eur J Clin Invest 34(12): 785-796

17. Caramori ML, Fioretto P, Mauer M (2006) Enhancing the predictive value of urinary albumin for diabetic nephropathy. J Am Soc Nephrol 17: 339-352

18. Wolf G, Ziyadeh FN (1999) Molecular mechanisms of diabetic renal hypertrophy. Kidney Int 56: 393-405.

19. Mahimainathan L, Das F, Venkatesan B, Choudhury GG (2006) Mesangial cell hypertrophy by high glucose is mediated by downregulation of the tumor suppressor PTEN. Diabetes 55: 2115-2125.

20. Shin SJ, Lee YJ, Tan MS, Hsieh TJ, Tsai JH (1997) Increased atrial natriuretic peptide mRNA expression in the kidney of diabetic rats. Kidney Int 51, 1100-1105.

21. Drummond K, Mauer M (2002) The early natural history of nephropathy in type 1 diabetes: II. Early renal structural changes in type 1 diabetes. Diabetes 51(5): 1580-1587.

22. White KE, Bilous RW (2000) Type 2 diabetic patients with nephropathy show structuralfunctional relationships that are similar to type 1 disease. J Am Soc Nephrol 11: 16671673. 
23. Tervaert TW, Mooyart AL, Amann K, Cohen AH (2010) Pathologic Classification of Diabetic Nephropathy. JASN (2) 4: 556-563.

24. Toyoda M, Najafian B, Kim Y, Caramouri ML, Mauer M (2007) Podocyte detachment and reduced glomerular capillary endothelial fenestration in human type 1 diabetic nephropathy. Diabetes 56(8): 2155-2160.

25. Siddiqi FA, Advani A (2013) Endothelial-Podocyte Crosstalk: The Missing Link Between Endothelial Dysfunction and Albuminuria in Diabetes. Diabetes 62 (11): 3647-3655.

26. Verzola D, Gandolfo MT, Ferrario F, Rastaldi MP, Villaggio B, Gianiorio F, Giannoni M, Rimoldi L, Lauria F, Miji M, Deferrari G, Garibotto G (2007) Apoptosis in the kidneys of patients with type II diabetic nephropathy. Kidney Int 72: 1262-1272.

27. Dalla Vestra M, Saller A, Mauer M, Floretto P (2001) Role of mesangial expansion in the pathogenesis of diabetic nephropathy. J Nephrol 14 Suppl 4:S51-S57

28. Kumar D, Zimpelmann J, Robertson S, Burns KD (2004) Tubular and interstitial cell apoptosis in the streptozotocin-diabetic rat kidney. Nephron Exp Nephrol 96: e77-e88.

29. Sanz AB, Santamaria B, Ruiz-Ortega M, Egido J, Ortiz A (2008) Mechanisms of renal apoptosis in health and disease. J Am Soc Nephrol 19: 1634-1642.

30. Maric C, Sandberg K, Hinojosa-Laborde C (2004) Glomerulosclerosis and tubulointerstitial fibrosis are attenuated with 17beta-estradiol in the aging Dahl salt sensitive rat. J Am Soc Nephrol 15: 1546-1556.

31. Benigni A, Zoja C, Campana M, Corna D, Sangalli F, Rottoli D, Gagliardini E, Conti S, Ledbetter S, Remuzzi G (2006) Beneficial effect of TGFbeta antagonism in treating diabetic nephropathy depends on when treatment is started. Nephron Exp Nephrol 104: e158-e168.

32. Mankhey RW, Bhatti F, Maric C (2005) 17beta-Estradiol replacement improves renal function and pathology associated with diabetic nephropathy. Am J Physiol Renal Physiol 288:F399-F405

33. Twigg SM, Cao Z, McLennan SV, Burns WC, Brammar G, Forbes JM, Cooper ME (2002) Renal connective tissue growth factor induction in experimental diabetes is prevented by aminoguanidine. Endocrinology 143: 4907-4915. 
34. Wahab NA, Yevdokimova N, Weston BS, Roberts T, Li XJ, Brinkman H, Mason RM (2001) Role of connective tissue growth factor in the pathogenesis of diabetic nephropathy. Biochem J 359: 77-87.

35. Umezono T, Toyoda $M$, Kato $M$, Miyauchi $M$, Kimura $M$, Maruyama $M$, Honma $M$, Yagame M, Suzuki D (2006) Glomerular expression of CTGF, TGF-beta 1 and type IV collagen in diabetic nephropathy. J Nephrol 19: 751-757.

36. Riser BL, deNichilo M, Cortes P, Baker C, Grondin JM, Yee J, Narins RG (2000) Regulation of connective tissue growth factor activity in cultured rat mesangial cells and its expression in experimental diabetic glomerulosclerosis. J Am Soc Nephrol 11: 25-38.

37. Park SK, Kim J, Seomun Y, Choi J, Kim DH, Han IO, Lee EH, Chung SK, Joo CK (2001) Hydrogen peroxide is a novel inducer of connective tissue growth factor. Biochem Biophys Res Commun 284:966-971.

38. Blom IE, van Dijk AJ, Wieten L, Duran K, Ito Y, Kleij L, deNichilo M, Rabelink TJ, Weening JJ, Aten J, \& Goldschmeding R (2001). In vitro evidence for differential involvement of CTGF, TGFbeta, and PDGF-BB in mesangial response to injury. Nephrol Dial Transplant 16: $1139-1148$.

39. Yokoi H, Mukoyama M, Sugawara A, Mori K, Nagae T, Makino H, Suganami T, Yahata K, Fujinaga Y, Tanaka I, Nakao K (2002) Role of connective tissue growth factor in fibronectin expression and tubulointerstitial fibrosis. Am J Physiol Renal Physiol 282: F933-F942.

40. McLennan SV, Kelly DJ, Cox AJ, Cao Z, Lyons JG, Yue DK, Gilbert RE (2002) Decreased matrix degradation in diabetic nephropathy: effects of ACE inhibition on the expression and activities of matrix metalloproteinases. Diabetologia 45: 268-275.

41. Metcalfe PD, Meldrum KK (2006) Sex differences and the role of sex steroids in renal injury. J Urol 176: 15-21.

42. Li SY, Huang PH, Yang AH, Tarng DC, Yang WC, Lin CC, Chen JW, Schmid-Schonbein G, Lin SJ (2014) Matrix metalloproteinase-9 deficiency attenuates diabetic nephropathy by modulation of podocyte functions and dedifferentiation. Kidney Int 86(2): 358-369. DOI: 10.1038/ki.2014.67. 
43. van der Zijl NJ, Hanemaaijer R, Tushuizen ME, Schindhelm RK, Boerop J, Rustemeijer C, Bilo HJ, Verheijen JH, Diamant M (2010) Urinary matrix metalloproteinase-8 and -9 activities in type 2 diabetic subjects: A marker of incipient diabetic nephropathy? Clin Biochem 43: 635-639.

44. Rysz J, Banach M, Stolarek RA, Pasnik J, Cialkowska-Rysz A, Koktysz R, Piechota M, Baj Z (2007) Serum matrix metalloproteinases MMP-2 and MMP-9 and metalloproteinase tissue inhibitors TIMP-1 and TIMP-2 in diabetic nephropathy. J Nephrol 20: 444-452.

45. Del PD, Anglani F, Forino M, Ceol M, Fioretto P, Nosadini R, Baggio B, Gambaro G (1997) Down-regulation of glomerular matrix metalloproteinase-2 gene in human NIDDM. Diabetologia 40: 1449-1454.

46. Romanic AM, Burns-Kurtis CL, Ao Z, Arleth AJ, Ohlstein EH (2001) Upregulated expression of human membrane type-5 matrix metalloproteinase in kidneys from diabetic patients. Am J Physiol Renal Physiol 281: F309-F317.

47. Skiles JW, Gonnella NC, Jeng AY (2001) The design, structure, and therapeutic application of matrix metalloproteinase inhibitors. Curr Med Chem 8: 425-474.

48. Brew K, Nagase H (2010) The tissue inhibitors of metalloproteinases (TIMPs): an ancient family with structural and functional diversity. Biochim Biophys Acta 1803: 55-71.

49. Sawai K, Mukoyama M, Mori K, Yokoi H, Koshikawa M, Yoshioka T, Takeda R, Sugawara A, Kuwahara T, Saleem MA, Ogawa O, Nakao K (2006) Redistribution of connexin43 expression in glomerular podocytes predicts poor renal prognosis in patients with type 2 diabetes and overt nephropathy. Nephrol Dial Transplant 21: 2472-2477.

50. Wright JA, Richards J, Becker DL (2012) Connexins and Diabetes. Cardiol Res and Prac: 2012:8 pages, DOI: 10.1155/2012/496904.

51. Ivaska J, Pallari HM, Nevo J, Eriksson JE (2007) Novel functions of vimentin in cell adhesion, migration, and signaling. Exp Cell Res 313: 2050-2062.

52. Satelli A, Li S (2011) Vimentin in cancer and its potential as a molecular target for cancer therapy. Cell Mol Life Sci 68: 3033-3046. 
